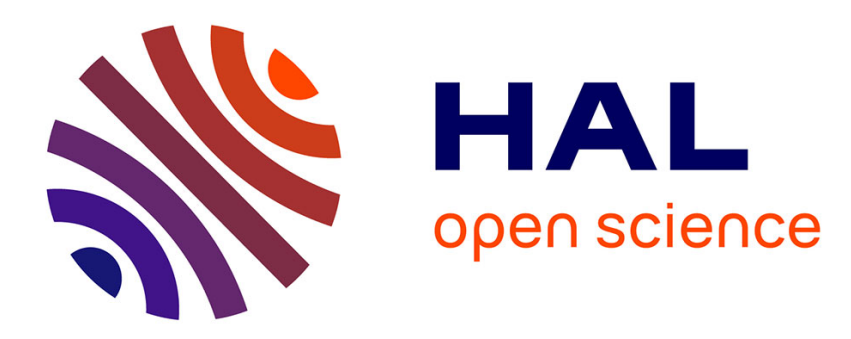

\title{
Computer-aided placement of air quality sensors using adjoint framework and sensor features to localize indoor source emission
}

\author{
Julien Waeytens, Sara Sadr
}

\section{- To cite this version:}

Julien Waeytens, Sara Sadr. Computer-aided placement of air quality sensors using adjoint framework and sensor features to localize indoor source emission. Building and Environment, 2018, 144, pp.184193. 10.1016/j.buildenv.2018.08.012 . hal-01862664

\section{HAL Id: hal-01862664 \\ https://hal.science/hal-01862664}

Submitted on 27 Aug 2018

HAL is a multi-disciplinary open access archive for the deposit and dissemination of scientific research documents, whether they are published or not. The documents may come from teaching and research institutions in France or abroad, or from public or private research centers.
L'archive ouverte pluridisciplinaire HAL, est destinée au dépôt et à la diffusion de documents scientifiques de niveau recherche, publiés ou non, émanant des établissements d'enseignement et de recherche français ou étrangers, des laboratoires publics ou privés. 


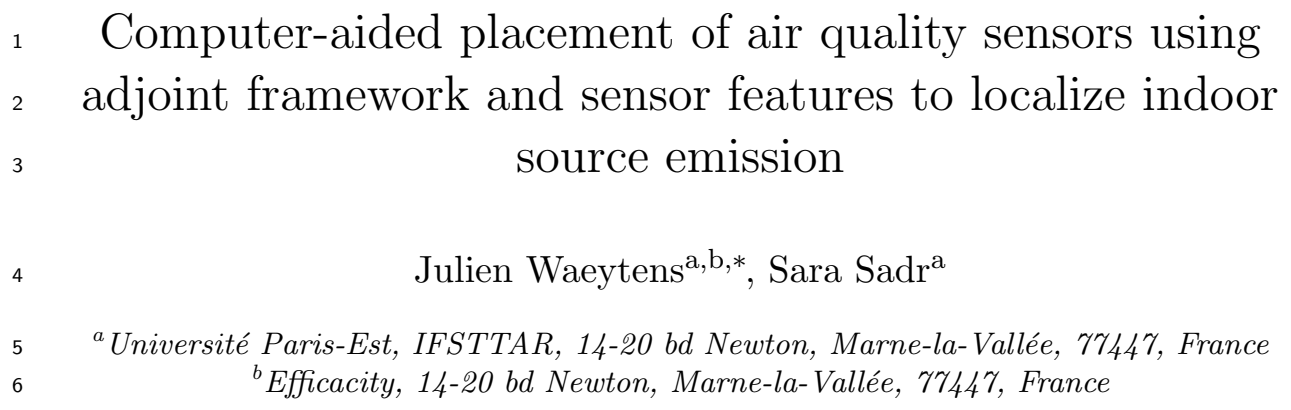

7 Abstract

8 With the improvement in sensor technologies, air quality is increasingly be-

9 ing monitored. Two major factors in obtaining relevant information are

10 the optimal placement and the number of air quality sensors. Moreover,

11 in cases of poor air quality, the information of the pollution level given by

12 the deployed sensors is not sufficient. An advanced understanding of the

13 data is required to precisely identify the source pollution and thus propose

14 effective solutions. In this article, a virtual testing strategy based on com-

15 putational fluid dynamics (CFD) is presented for the optimal placement of

16 indoor air quality sensors. We determine the placement of sensors in view

17 of localizing the maximum of sources emitting on the indoor environment

18 surfaces. Therefore, an adjoint framework is used to obtain the observable

19 region associated with a given sensor position. The proposed method takes

20 into account technical sensor features, such as the limit of detection (LOD).

21 Two applications are studied: a simple 2D case and a real $3 \mathrm{D}$ room. In

22 these examples, we first show that reducing the LOD of the sensors by one

23 order of magnitude can increase the observable area by more than $50 \%$.

${ }^{*}$ Corresponding author: E-mail: julien.waeytens@ifsttar.fr (J. Waeytens), Ph: +331 816684 53, Fax: +33 1816680 01, Postal address: Cité Descartes, 14-20 bd Newton, F-77447 Marne-la-Vallée cedex 2, France 
Then, we note that one-fourth of the potential sensor placements observe almost nothing and that $80 \%$ of the potential sensor placements have an observable area two times smaller than the optimal sensor position determined by the proposed CFD-based strategy.

44 in sensor technologies, notably nanotechnologies. Hence, the gas sensors

45 become cheaper, smaller, more sensitive, less energy-consuming, etc... To 
46 get more details on low-cost sensors for air quality purposes, the reader can 47 refer to the review article [8]. The localization of VOC sources can also 48 be useful for the preservation of cultural heritage, notably artwork, and for 49 structural health monitoring purposes. In most regions of France, the presence of woodborers, such as termites, has harmful effects on the safety of structures. The VOC chemical signature of termites can be used for their early detection and localization, which will provide the ability to limit the 53 use of termiticides and to preserve the structure.

54

To efficiently monitor air quality, the number of sensors and their positioning are crucial. In most measurement campaigns, the gas sensors are placed in an empirical way. For example, in a room, an air quality sensor is usually positioned at the breathing zone height or approximately $0.5 \mathrm{~m}$ from the ceiling in the middle of the room. Unfortunately, this placement does not take into account the characteristics of the room, i.e. the geometry and the ventilation. As a consequence, bad sensor placement may lead to the nondetection of some sources. To well-position gas sensors, we can take advantage of numerical simulations derived from physical models. In indoor air quality applications, the gas concentration can be predicted using multizone $[9 ; 10 ; 11 ; 12]$ and CFD $[9 ; 13 ; 14]$ models. Multizone techniques, which provide the time evolution of the averaged concentration in each zone as output, are easy to use and run on a standard laptop. Nevertheless, they consider strong hypotheses, such as a well-mixed concentration. With the ongoing improvement of computers and numerical methods, CFD approaches appear to be promising for the prediction of indoor air quality and for optimal sensor placement. In fact, CFD provides a fine description of the spatial concentration in the indoor environment, but the computations are time 
consuming. A good compromise to study the indoor air quality of an entire building would be to couple multizone and CFD models, as proposed in [15]. To the best of the authors' knowledge, few publications have addressed the optimal placement of gas sensors for IAQ applications. The design of an optimal sensor network, i.e. the number and positioning of sensors, has been studied in greater depth in terms of chemical and biological warfare (CBW) and transmission of infectious diseases (TID). The sensor positions are chosen to early detect and localize indoor contamination. Different methods aim to maximize the coverage area of sensors and to minimize the response time for various sets of release scenarios. In [16], the sensor coverage area is evaluated using CFD and an adjoint advection-diffusion equation, whereas physical model-free approaches based on a dynamical systems approach are preferred in [17]. Note that the adjoint framework is a useful numerical tool for various applications. First, it provides, at a low computational cost, the functional gradient and the Hessian matrix involved in inverse calculations to update the parameters of fluid mechanics models [18; 19] and to reconstruct the concentration fields $[20 ; 21 ; 22]$. Additionally, it is used in sensitivity analyses to study the influence of physical model parameters on a quantity of interest $[23 ; 24]$. The adjoint framework is also considered for estimating the modeling or discretization error on a quantity of interest $[25 ; 26 ; 27]$.

Once the positions of the sensors are fixed, knowledge of the concentration given by the deployed sensors is not sufficient for proposing efficient solutions for indoor air quality improvement or for localizing woodborers. One needs to localize and to quantify the source emissions. To achieve this purpose, two families of methods can be found in the literature, i.e. data- 
driven methods and physical model-based methods. Direct measurements of the source emissions on different surfaces of the environment (furniture, wall, floor, door, etc.) can be planned using innovative sensors, such as fibers placed in a specific device for on-site emission control [28;9]. This method enables accurate in situ quantification of the source emissions for building materials and furniture, but it requires a large number of sensor devices. Another data-driven method to evaluate source emissions is indirect measurements. In contrast to the previous methods, the air quality sensors are placed in the room volume and not directly on a surface. Databases of the chemical signatures of sources and a priori information of the studied environment collected via questionnaire, including the type and the age of the building materials, renovations, cleaning products and ventilation, are commonly considered in these methods. Finally, the sensor outputs associated with various chemical compounds are analyzed via statistical tools, such as proper component analysis and linear regression, to identify the source emissions $[4 ; 5 ; 29 ; 30]$. In practice, the chemical compounds emitted by some items in the studied environment may not be referenced in a database. Consequently, these methods may only approximately identify the sources. Physical model-based approaches via inverse modeling techniques can also be valuable for the localization and the quantification of source emissions. In general, inverse problems that couple model and sensor outputs are not well-posed in the sense of Hadamard, i.e the existence, uniqueness and nonhigh sensitivity of the solution to the sensor outputs. To address this issue, a sufficient number of well-positioned sensors is required, and regularization must be considered in the mathematical formulation of the inverse problem. In deterministic settings, Tikhonov regularization is commonly considered and consists of adding penalization terms to the data misfit functional, as 
discussed in $[15 ; 31]$ for convective-diffusive transport source inversion. In probabilistic inversion formalism, notably Bayesian model updating, which was applied in [32] for $\mathrm{CO} 2$ regional source estimations, the model parameter probability distributions are interesting on two counts. They ensure the problem regularization and provide a confidence interval on the identified source emissions. Nevertheless, probabilistic inversions can be much more time consuming than deterministic ones. Finally, the adjoint framework, previously mentioned for the optimal placement of sensors, can also be used for source localization, as shown in $[33 ; 15]$.

In the present article, we propose a virtual testing strategy, taking into account the specificities of the indoor environment (geometry and ventilation) via CFD and gas sensor features (limit of detection), to efficiently select the number and positions of sensors to localize indoor sources. We define the "optimal sensor placement" as the combination of gas sensors that maximizes the coverage area. The authors showed in previous works [21] that the sensor observable area can be computed at a reasonable cost using the adjoint framework. Herein, we emphasize that the coverage area can be increased not only by adding sensors but also by using sensors with a lower limit of detection. The rest of this article is organized as follows. In Section 2.1, a physical direct model to predict the gas dispersion is presented. Then, we define the adjoint equations in Section 2.2 and introduce a new adjointbased criterion integrating sensor features to evaluate the observable area of potential sensor positions in Section 2.3. An overview of the optimal sensor placement strategy is given in Section 2.4, and it is applied to a $2 \mathrm{D}$ case and a real $3 \mathrm{D}$ room in the last section. 
165

\section{Materials \& Methods}

\subsection{Simulation of pollutant propagation - Direct problem}

To predict the dispersion of gas, advection-diffusion-reaction models are commonly used $[9 ; 13 ; 14]$. As a first step, we consider non-reactive gases, i.e. reaction phenomena are not modeled. Hence, the cartography of the gas concentration in a two- or three-dimensional space domain $\Omega$ is obtained from the advection-diffusion model. Four types of boundaries can be distinguished. A boundary presenting a known prescribed concentration $C_{p}$ is denoted $\partial_{p} \Omega$. Potential pollution emissions, to be precisely located by the optimal placement of gas sensors, are on the boundary $\partial_{u} \Omega$, whereas a boundary that does not present source emission is $\partial_{n} \Omega$. Lastly, $\partial_{o} \Omega$ denotes the outgoing flow boundary.

The pollutant concentration $C(\mathbf{x}, t)$ in the domain $\Omega \subset \mathbb{R}^{n}, n \in\{2,3\}$ can be obtained by solving the unsteady advection-diffusion model, which is also called the "direct problem",

$$
\left\{\begin{array}{l}
\frac{\partial C}{\partial t}(\mathbf{x}, t)+\mathbf{v}(\mathbf{x}, t) \cdot \nabla C(\mathbf{x}, t)-\nu(\mathbf{x}, t) \Delta C(\mathbf{x}, t)=0 \quad \text { in } \Omega \times[0, T] \\
C(\mathbf{x}, t)=C_{p}(\mathbf{x}, t) \quad \text { on } \partial_{p} \Omega \times[0, T] \\
C(\mathbf{x}, t)=C_{u}(\mathbf{x}, t) \quad \text { on } \partial_{u} \Omega \times[0, T] \\
\nabla C(\mathbf{x}, t) \cdot \mathbf{n}=0 \quad \text { on } \partial_{n} \Omega \times[0, T] \\
\nabla C(\mathbf{x}, t) \cdot \mathbf{n}=0 \quad \text { on } \partial_{o} \Omega \times[0, T] \\
C(\mathbf{x}, t=0)=C_{0}(\mathbf{x}) \quad \text { in } \Omega
\end{array}\right.
$$

In Eq. (1), $\mathbf{v}$ is the flow velocity, $\nu$ denotes the diffusion parameter, which is the sum of the molecular and turbulent diffusion, and $\mathbf{n}$ denotes the outside 
normal vector to the surface.

When the flow and the source emission can be considered stationary with respect to the monitoring time, the concentration field $C(\mathbf{x})$ can be obtained at a lower computation cost using a steady advection-diffusion model

$$
\left\{\begin{array}{l}
\mathbf{v}(\mathbf{x}) \cdot \nabla C(\mathbf{x})-\nu(\mathbf{x}) \Delta C(\mathbf{x})=0 \quad \text { in } \Omega \\
C(\mathbf{x})=C_{p}(\mathbf{x}) \quad \text { on } \partial_{p} \Omega \\
C(\mathbf{x})=C_{u}(\mathbf{x}) \quad \text { on } \partial_{u} \Omega \\
\nabla C(\mathbf{x}) \cdot \mathbf{n}=0 \quad \text { on } \partial_{n} \Omega \\
\nabla C(\mathbf{x}) \cdot \mathbf{n}=0 \quad \text { on } \partial_{o} \Omega .
\end{array}\right.
$$

For example, Eq. (2) can be used to model the dispersion of moisture or woodborers emissions during a measurement campaign under mastered air flow conditions, e.g. when the indoor occupants have left. In the following, we limit our study to stationary cases.

\subsection{Sensitivity area of a gas sensor - Adjoint problem}

Physically, the solution of the adjoint problem corresponds to a sensitivity function in terms of a quantity of interest. Hence, to obtain the sensor observable area, we choose the gas concentration at the sensor location $\mathbf{x}_{\mathbf{s}}$ as the quantity of interest. It is given by

$$
J=\int_{\Omega} f_{s}\left(\mathbf{x}-\mathbf{x}_{\mathbf{s}}\right) C(\mathbf{x}) d \Omega
$$

5 where $f_{s}$ is a space function to extract the gas concentration at the sensor location $\mathbf{x}_{\mathbf{s}}$. In practice, we can take:

$$
f_{s}\left(\mathbf{x}-\mathbf{x}_{\mathbf{s}}\right)=\left\{\begin{array}{l}
1 /\left|\Omega_{s}\right| \text { for } \mathbf{x} \in \Omega_{s} \\
0 \text { elsewhere. }
\end{array}\right.
$$


The domain $\Omega_{s}$ is a sphere of radius $R_{s}$ centered at the sensor location $\mathbf{x}_{\mathbf{s}}$.

From the quantity of interest, we introduce the adjoint problem (5) and compute its numerical solution $\tilde{C}$.

$$
\left\{\begin{array}{l}
-\mathbf{v}(\mathbf{x}) \cdot \nabla \tilde{C}(\mathbf{x})-\nu(\mathbf{x}) \Delta \tilde{C}(\mathbf{x})=f_{s}\left(\mathbf{x}-\mathbf{x}_{\mathbf{s}}\right) \quad \text { in } \Omega \\
\tilde{C}(\mathbf{x})=0 \quad \text { on } \partial_{p} \Omega \\
\tilde{C}(\mathbf{x})=0 \quad \text { on } \partial_{u} \Omega \\
\nabla \tilde{C}(\mathbf{x}) \cdot \mathbf{n}=0 \quad \text { on } \partial_{n} \Omega \\
\nu \nabla \tilde{C}(\mathbf{x}) \cdot \mathbf{n}+\mathbf{v}(\mathbf{x}) \cdot \mathbf{n} \tilde{C}(\mathbf{x})=0 \quad \text { on } \partial_{o} \Omega
\end{array}\right.
$$

Note that the adjoint problem (5) is a backward-advection-diffusion problem with a source emission located at the sensor position. This adjoint problem can be solved with the same CFD software as that used for the direct problem. For greater detail on the derivation of the adjoint problem, the reader can refer to $[21]$.

\subsection{Computation of sensor observable area - A new adjoint-based criterion}

After defining the adjoint problem, we propose an adjoint-based criterion (6) that takes into account the sensor features, i.e., the LOD of the gas sensor, in view of obtaining the sensor observable area.

$$
|\nabla J| \frac{A_{s} S}{d I_{m}}>1
$$

where:

- $J$ (resp., $\nabla J)$ is the functional (resp., functional gradient) associated with the gas concentration at the sensor location $\mathbf{x}_{\mathbf{s}}$ defined in Eq. (3)

- $A_{s}$ is the minimum source area expected to be localized 
- $S$ is the order of magnitude of the source emission

- $d I_{m}$ is the limit of detection of the gas sensor

The sensitivity of the gas concentration at the sensor location $\mathbf{x}_{\mathbf{s}}$ to the surface source emissions, which corresponds to the functional gradient $\nabla J$, can be evaluated using the adjoint framework. Following the method in [21], we can show that:

$$
\nabla J(\mathbf{x})=\nu(\mathbf{x}) \nabla \tilde{C}(\mathbf{x}) \cdot \mathbf{n}
$$

where $\mathbf{n}$ denotes the unit outer normal vector along the surface.

In summary, the observable area of a gas sensor located at a given position $\mathbf{x}_{\mathbf{s}}$ can be numerically predicted by

$$
\mathbf{x} \in \partial_{u} \Omega \text { such that }|\nu(\mathbf{x}) \nabla \tilde{C}(\mathbf{x}) \cdot \mathbf{n}| \frac{A_{s} S}{d I_{m}}>1 .
$$

Let us physically interpret the different terms in the proposed criterion (8). The first part $|\nabla J(\mathbf{x})|$ takes into account the sensor position $\mathbf{x}_{\mathbf{s}}$ and gives the sensitivity map of the gas sensor output to the surface source emission. It is numerically obtained from the solution $\tilde{C}$ of the adjoint problem defined in Eqs. (5). In [21], we proved that a null value of $|\nu(\mathbf{x}) \nabla \tilde{C}(\mathbf{x}) \cdot \mathbf{n}|$ on a boundary $\partial_{b} \Omega \subset \partial_{u} \Omega$ implies that potential source emissions on $\partial_{b} \Omega$ cannot be detected by a sensor at the position $\mathbf{x}_{\mathbf{s}}$.

The new contribution in this article concerns the next two terms. The second term $A_{s} \times S$ relies on a priori information of the source emissions that are expected to be detected. If we are interested in emissions on large surfaces, such as painted walls, $A_{s}$ should be approximately a few tens of square meters. By contrast, if we are interested in emissions on small surfaces, such as furniture, $A_{s}$ should be approximately one square meter. A small value 
of $A_{s}$, i.e. less than one square meter, can also be useful for the early detection of termites. The order of magnitude of potential emissions is taken into account with the parameter $S$. For formaldehyde furniture emission it can be higher than 1ppm [34] whereas it is a hundred times lower for VOCs emitted by molds [35]. In the proposed criterion (8), the observable area for a given positioned sensor depends on the product $A_{s} \times S$. Hence, the higher this product is, the larger the observable area.

Lastly, the sensor detection limit, depending on technology features, corresponds to the third term in the proposed criterion (8). In the Results Section, we show how the observable area increases as the limit of detection of the sensors decreases.

\subsection{Outline of the virtual testing strategy for the optimal placement of air} quality sensors

This section aims to present the steps in computer-aided sensor placement. The process is summarized in Figure 1.

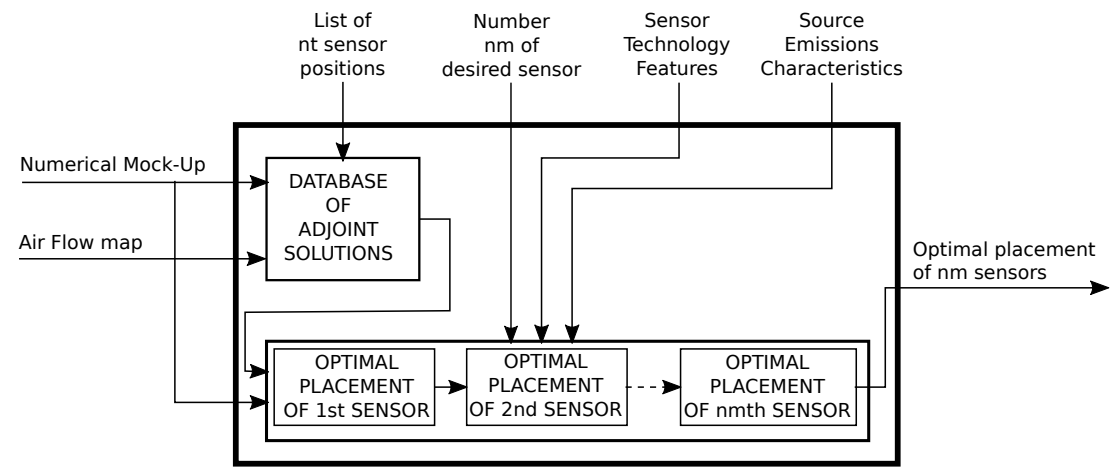

Figure 1: Architecture of the computer-aided method for the optimal placement of air quality sensors 
The proposed strategy necessitates a mock-up of the studied environment and a fine description of the air flow. The air flow map can be obtained from experiments, empirical models or computational fluid dynamics. Let us emphasize that [22] previously pointed out that a rough approximation of the real flow can lead to a non-representative concentration simulation. Thus, special attention must be given to obtaining the air flow map; otherwise, the proposed placement of gas sensors can be incorrect. After defining a list of $n_{t}$ potential sensor positions, we solve the adjoint problem (5) associated with each sensor position. All the $n_{t}$ adjoint solutions are stored in a database. Note that this step is fully parallelizable and is performed only once in a off-line stage.

In the proposed virtual testing strategy, the observable area is computed for the $n_{t}$ sensor positions. As shown in the previous section, the observable area is obtained from the adjoint-based observable criterion (8). In addition to the adjoint solution, a priori information of the sensor technology is also required, i.e. the limit of detection $d I_{m}$ of the sensor and the source to be localized, i.e. the orders of magnitude of area $A_{s}$ and level $S$ of the source emissions. Lastly, the optimal placement corresponds to the one with the largest observable area. When the number $n_{m}$ of desired sensors is strictly greater than one, the optimal placement is performed in a hierarchical manner. We start by optimally placing the first sensor and fix its position; then, we seek the optimal placement of the second sensor and fix its position, and so on. As practical outputs for the users, the computer-aided method provides, as a visualization on the numerical mock-up, the observable area of each selected sensor position and the coverage area in square meters for each sensor and for the combination of all $n_{m}$ sensors. 


\section{Results}

\subsection{Application 1-2D simple problem}

To gain a better understanding, let us first consider a $2 \mathrm{D}$ academic problem (see Figure 2). The $2 \mathrm{D}$ domain $\Omega$ is a square with $10 \mathrm{~m}$ sides, and the flow $\mathbf{V}$ is uniform. The velocity amplitude (resp. the velocity orientation angle) is $1 \mathrm{~m} / \mathrm{s}$ (resp., $27^{\circ}$ ), and the diffusion parameter $\nu$ is $2.2 \times 10^{-2} \mathrm{~m}^{2} / \mathrm{s}$ which corresponds to the order of magnitude of the turbulent diffusion. As introduced in Section 2.1, $\partial_{o} \Omega$ denotes the outgoing flow boundary. In this example, we aim to optimally place gas sensors to localize and quantify sources coming from the boundary $\partial_{u} \Omega$. We focus on the detection of a source emitting on an area greater than $1 m$ on $\partial_{u} \Omega$ and whose order of magnitude of the amplitude is approximately 100ppm. From this information, we take $A_{s}=1 \mathrm{~m}$ and $S=100 \mathrm{ppm}$ in the observable criterion (8). Moreover, in the domain $\Omega$, gas sensors can be placed at a limited number of positions. The sixteen potential sensor positions are shown in Figure 2 .
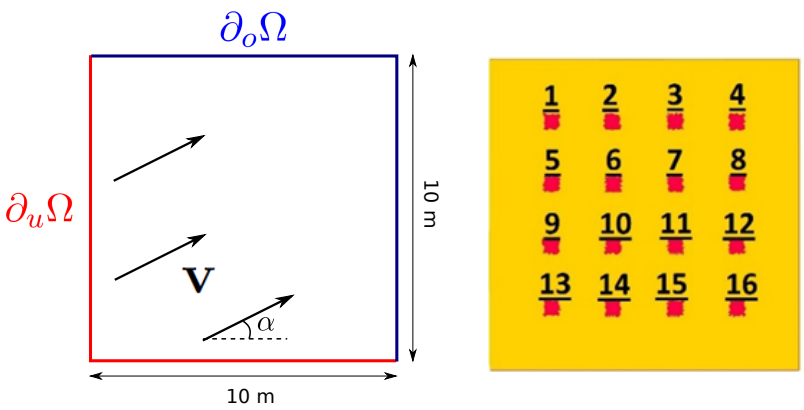

Figure 2: Geometry of the 2D problem (left) and potential positions of the gas sensor (right) 
the observable area is studied for a unique given sensor. Then, the virtual testing strategy is illustrated to optimally place several sensors.

3.1.1. Influence of the $L O D$ on the observable area for a given sensor position

In this section, we consider a given sensor position, that is, Sensor \#3 (see Figure 2). The objective is to evaluate its observable area for different LODs of the gas sensor. We use the proposed adjoint-based criterion (8). First, one needs to solve the adjoint problem defined in Eq. (5), which corresponds to a backpropagation of a pollutant emitted at Sensor location \#3 (see Figure 3). Then, from the adjoint solution $\tilde{C}$ and the LOD $d I_{m}$, we compute the criterion (8) and deduce the observable area associated with the considered sensor. In Figure 3, we present the adjoint field $\tilde{C}$ and the observable area of Sensor \#3 for two different LODs: 10ppm and 0.1ppm. For the considered flow, source emissions on the bottom edge cannot be detected by Sensor \#3. The observable area is located around the middle of the left edge. Its precise position along the left edge is $4.9 m \pm 1.1 m$ for an LOD of $10 p p m$ and $4.9 m \pm 2.2 m$ for an LOD of $0.1 p p m$.

The evolution of the observable area for a wide range of LODs is presented in Figure 4. As expected, a reduction in the LOD leads to an increase in the observable area. The observable area for Sensor \#3 is one and a half times larger (resp. two times larger) when using a gas sensor with a $1 \mathrm{ppm}$ LOD (resp., 0.1ppm LOD) than one with a 10ppm LOD. In summary, this study shows that the LOD of the gas sensor has a strong impact on the observable area for detecting source emissions. Consequently, the LOD must be considered in the optimal placement strategies of air quality sensors. 


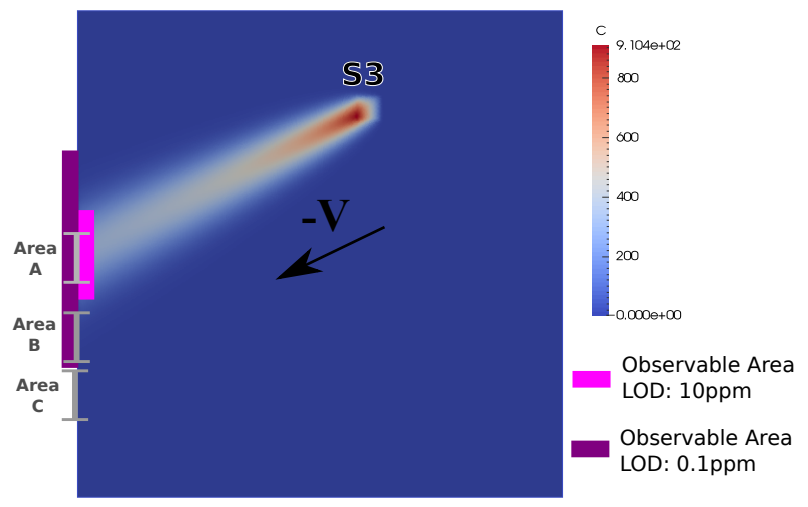

Figure 3: Adjoint problem solution $\tilde{C}$ associated with Sensor \#3 and its observable area for an LOD of $10 \mathrm{ppm}$ and $0.1 \mathrm{ppm}$ - Definition of Source Areas A, B, C for numerical validation of the observable criterion

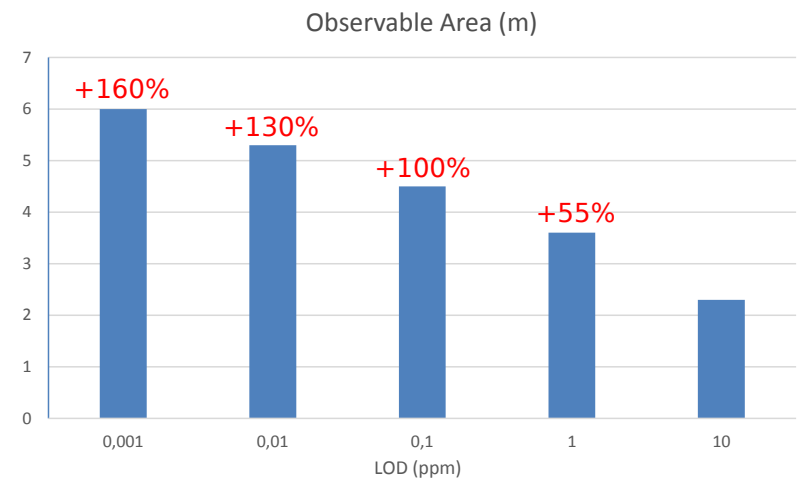

Figure 4: Observable Area of Sensor 3 as a function of the LOD - The observable area for an LOD of $0.1 \mathrm{ppm}$ is $100 \%$ larger than that with an LOD of $10 \mathrm{ppm}$

On the basis of the adjoint-based criterion (8), we were able to evaluate the observable area associated with a given sensor position. Let us numerically validate this observable criterion. Sensor position \#3 is still considered, and three source locations are defined on the left edge of the $2 \mathrm{D}$ 
domain (see Figure 3). The different sources are 1 meter in length, and their amplitude is 100ppm. As predicted by the proposed virtual testing strategy, a source in Area A can be detected by the sensor with both LODs (0.1ppm and 10ppm), a source emitted in Area B can be detected only by the sensor with an LOD of $0.1 \mathrm{ppm}$, and neither the $0.1 \mathrm{ppm}$ LOD sensor nor the 10ppm LOD sensor can detect a source in Area C. For each source, we simulate the associated gas dispersion by solving the direct advection-diffusion equations (2) and obtain the gas concentration at Sensor position \#3. From this concentration, we can verify whether the source is detected by the sensors with an LOD of $0.1 p p m$ or 10ppm. In Table 1 , the results show that the sensor observable area is well predicted by the adjoint-based criterion (8). A 100ppm source emitted in Area B leads to a gas concentration of 3.27ppm at Sensor position \#3. This concentration can be detected by the $0.1 \mathrm{ppm}$ LOD sensor but not by the 10ppm LOD sensor. This result is in agreement with the predicted observable area shown in Figure 3.

\begin{tabular}{|c|c|c|c|}
\hline \multirow{2}{*}{ Source location } & Concentration & $\begin{array}{r}\text { Source detected by the sensor ? } \\
\text { (Y: Yes, N: No) }\end{array}$ \\
\cline { 3 - 4 } & at sensor position \#3 & $0.1 p p m$ LOD & $10 p p m$ LOD \\
\hline Area A $(4.9 m \pm 0.5 m)$ & $56.06 p p m$ & $\mathbf{Y}$ & $\mathbf{Y}$ \\
\hline Area B $(3.3 m \pm 0.5 m)$ & $3.27 p p m$ & $\mathbf{Y}$ & $\mathrm{N}$ \\
\hline Area C $(2.2 m \pm 0.5 m)$ & $0.04 p p m$ & $\mathrm{~N}$ & $\mathrm{~N}$ \\
\hline
\end{tabular}

Table 1: Numerical validation of the adjoint-based observable criterion - Concentration at Sensor position \#3 simulated for different source locations and verification of the source detection for a $0.1 p p m$ LOD sensor and a 10ppm LOD sensor 


\subsubsection{Optimal placement of gas sensors considering a fixed $L O D$}

The optimal placement of the gas sensors is achieved using the virtual testing strategy presented in Section 2.4. First, we determine the optimal placement of the first sensor. Hence, for each sensor position, the associated adjoint problem is solved and saved in a database. The use of the adjoint solutions and the LOD in criterion (8) enable the evaluation of the observable area of each sensor. Herein, the LOD is fixed to 10ppm. The sensor position with the largest observable area is selected as the "optimal placement". The observable area associated with each sensor is summarized in Figure 5. Note that the sum of the observable areas on the bottom and left edges corresponds to the total observable area on the boundary $\partial_{u} \Omega$. We observe that Sensors \#1 to \#11 can detect a source emission only on the left edge, whereas Sensors \#12 and \#14 are able to detect a source emission located in the lower-left corner of the domain involving the left and bottom edges. Among the 16 potential positions, 13 positions have a total observable area between 1 and $2.5 \mathrm{~m}$, and only 3 positions give an observable area larger than $3 \mathrm{~m}$. Sensor \#12, which has a total observable area slightly larger than those of Sensor \#15 and Sensor \#16, is selected as the optimal placement.

As mentioned in Figure 1, the placement of several air quality sensors is performed in a hierarchical manner. After finding the optimal placement of the first sensor, we fix this sensor and determine the optimal placement of the second sensor, and so on. Previously, Sensor \#12 was determined as the optimal placement for the first sensor. To determine the optimal placement of several sensors, we evaluate the observable areas (see Figure 5 ) and select the sensor combination with the highest total observable area. The combination of Sensors \#12 and \#16 gives the highest total observable 


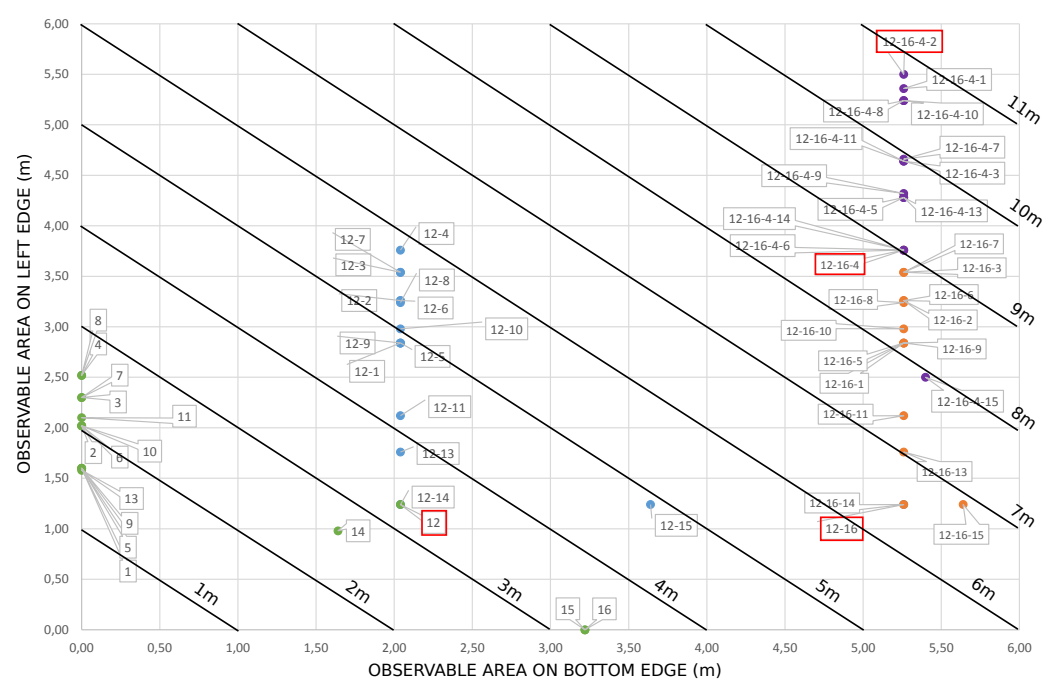

Figure 5: Optimal placement (red rectangles) in the 2D domain of several sensors of with an LOD of 10ppm - Observable Areas - Isovalues of the total observable area are represented as solid lines

area, that is, $6.5 \mathrm{~m}$. We can see that adding Sensor \#16 improves the observable area on only the bottom edge, increasing from $2 \mathrm{~m}$ to $5.5 \mathrm{~m}$. The observable area on the left edge is increased by the optimal placement of three sensors, i.e., Sensors \#12, \#16 and \#4. We note that the the total observable area is three times larger for the optimal placement of four sensors $(\# 12, \# 16, \# 4, \# 2)$ than for the optimal placement of a single sensor $\# 12$.

\subsubsection{Optimal placement of several sensors considering different $L O D$}

In Section 3.1.1, we showed that the LOD has a significant influence on the observable area for detecting source emissions. As a consequence, two factors can be investigated to improve the observable area: the number of gas sensors and the LOD. In this section, we study the optimal placement 
376

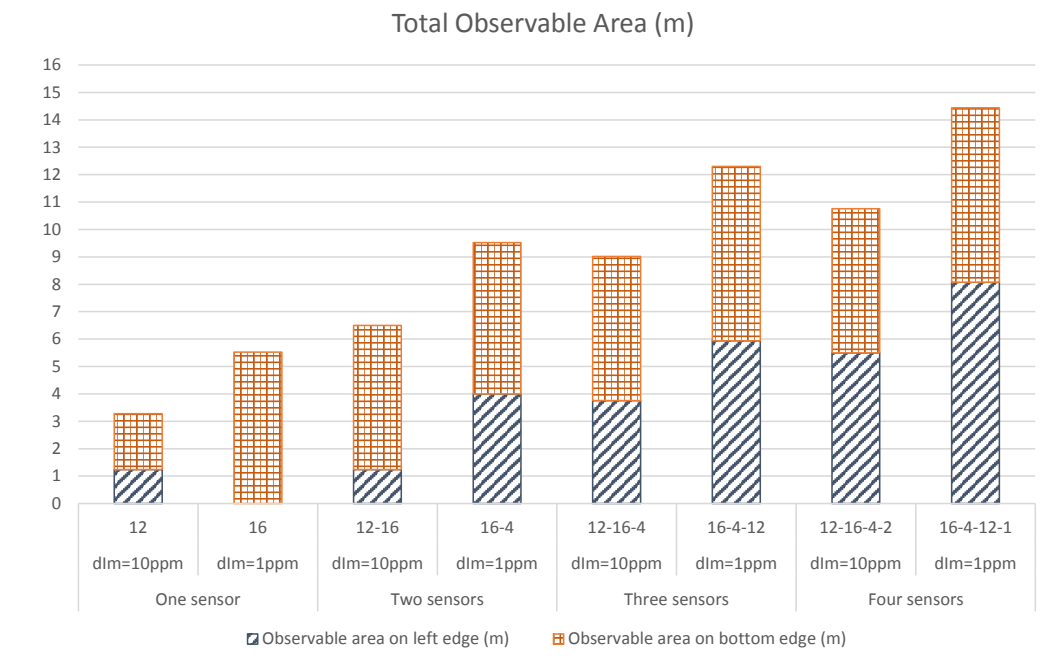

Figure 6: Evolution of the observable area as a function of the number of sensors and the LOD (10ppm and 1ppm)

of several sensors in the $2 \mathrm{D}$ domain when considering sensors with an LOD of either $10 \mathrm{ppm}$ or $1 \mathrm{ppm}$. The results are summarized in Figure 6. We observe that the optimal positions of gas sensors may differ according to the LOD. At a 10ppm LOD, Sensor \#12 is selected as optimal, whereas Sensor \#16 is optimal at a 1ppm LOD. Note that Sensor \#16 with a 1ppm LOD has a total observable area that is approximately twice the size of that of Sensor \#12 with a 10ppm LOD. To reach a total observable area of $9 \mathrm{~m}$, we can either use two Sensors (\#16,\#14) with a 1ppm LOD or three Sensors (\#12, \#16,\#4) with a 10ppm LOD.

In Figure 7, we present the observable area associated with the optimal placement of four gas sensors with LODs of either 10ppm or $1 \mathrm{ppm}$. The coverage is disparate on $\partial_{u} \Omega$ for a $10 \mathrm{ppm}$ LOD while it is widespread for a $1 \mathrm{ppm}$ 
LOD. Nevertheless, for both cases, source emissions cannot be detected in the upper part of the left edge and the right part of the bottom edge due to the considered flow $\mathbf{V}$ and the distribution of the sixteen potential sensor positions. Lastly, for the optimal placement with 1ppm LOD gas sensors, the observable area of some sensors overlaps, notably Sensors \#12 and \#16. Thus, a source emitted in the overlapped region would be detected by both Sensors \#12 and \#16.
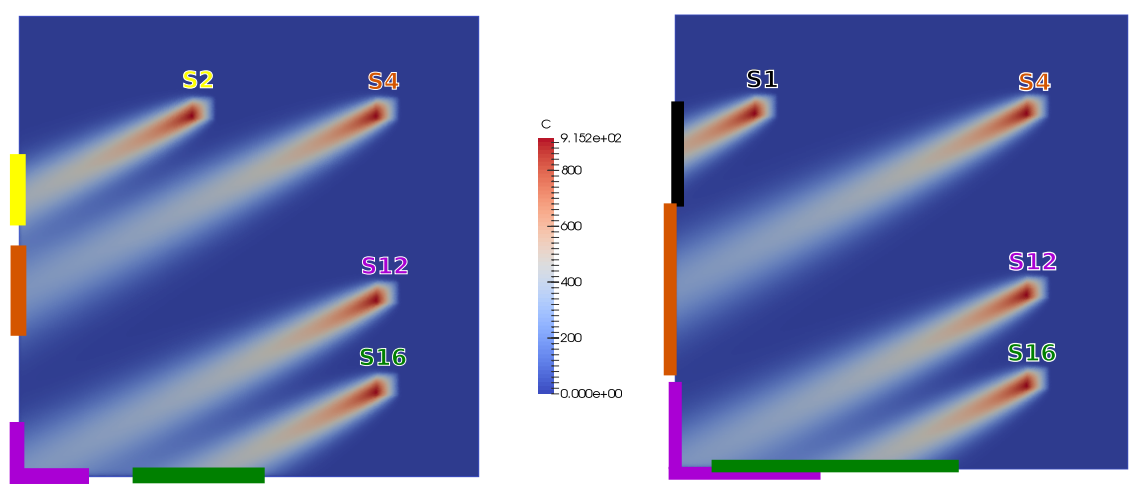

Figure 7: Map of the observable area for the optimal placement of 4 sensors with LODs of $10 \mathrm{ppm}$ (left) and of 1 ppm (right)

\subsection{Application 2 - 3D laboratory room}

In this section, we illustrate the computer-aided method for the optimal placement of gas sensors in a real 3D laboratory room, including furniture and ventilation systems, located at the IFSTTAR research institute. The dimensions of the room are $5.9 m \times 6.2 m \times 4.2 m$, which correspond to a volume of $150 \mathrm{~m}^{3}$. As mentioned in Figure 1, we first need a numerical mockup and indoor air flow map (see Figure 8). For that, the incoming flows from the heating duct, the two ventilation grids and the door were measured using 


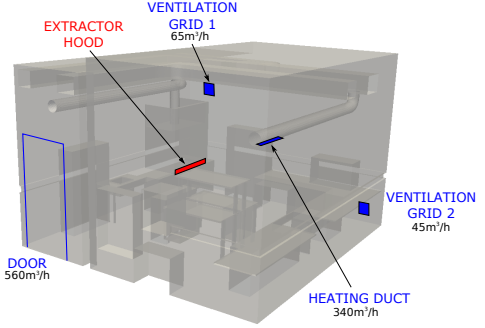

a 1D hot wire anemometer, and the values are reported in Figure 8. Note that the flow exits only from the extractor hood. From this information and the numerical mock-up, we simulate the stationary turbulent flow using the $k-\omega S S T$ Reynolds Average Navier-Stokes (RANS) model in the CFD software "Code_Saturne" [36]. In Figure 8, we can see that the airflow entering from the contour of the door is highly turbulent in the vicinity of the door and that a portion of it goes straight in the direction of the extractor hood. In terms of the incoming flow from the second ventilation grid, the main portion circulates close to the ground between the wall and the furniture. Lastly, the velocity flow from the heating duct oriented in the z-direction impacts the top of the furniture immediately below, which generates flow recirculation. From the RANS turbulent flow simulation, let us note that we also evaluate the turbulent diffusion involved in the diffusion parameter $\nu$. A practical rule of thumb consists to compute the turbulent diffusion $\nu_{t}$ from the turbulent viscosity $\mu_{t}$ and the density $\rho$ by $\nu_{t}=\left(1 / S c_{t}\right) \mu_{t} / \rho$ where the turbulent Schmidt number $S c_{t}$ is taken to 0.7.

Figure 8: Numerical mock-up of the laboratory room and measured incoming flows (left)

- Flow simulated by CFD software (right)

${ }_{411}$ In the laboratory room, the potential sensor positions presented in Fig- 
ure 10 are equally distributed every $50 \mathrm{~cm}$ at three heights above the ground, namely, $0.5 \mathrm{~m}, 1 \mathrm{~m}$ and $1.5 \mathrm{~m}$. There are 121 sensor positions per height, for a total of 363 potential sensor positions. The potential sensor positions are shown in Figure 10. Herein, we aim to select the sensor positions that produce the maximum observable on all the lateral surfaces (door face, furniture face, extractor hood face, back face). To evaluate the observable area, we compute the adjoint concentration $\tilde{C}$ associated with each sensor position and store the values in a database. In practice, the adjoint problems (5) are solved using the Streamline Upwind Petrov-Galerkin (SUPG) formulation [37] in the finite element code "FreeFem++" [38]. In Figure 9, we present the adjoint solution associated with Sensor position \#86, which is located close to the furniture wall $0.5 \mathrm{~m}$ above the floor. The adjoint field $\tilde{C}$ being a sensitivity function of the concentration at the sensor position to source emissions, Figure 9 shows that a gas sensor at position \#86 is sensitive to sources on a part of the furniture face and of the back face. Thus, a single sensor at position \#86 may not be able to detect source emissions on the extractor hood and door faces.
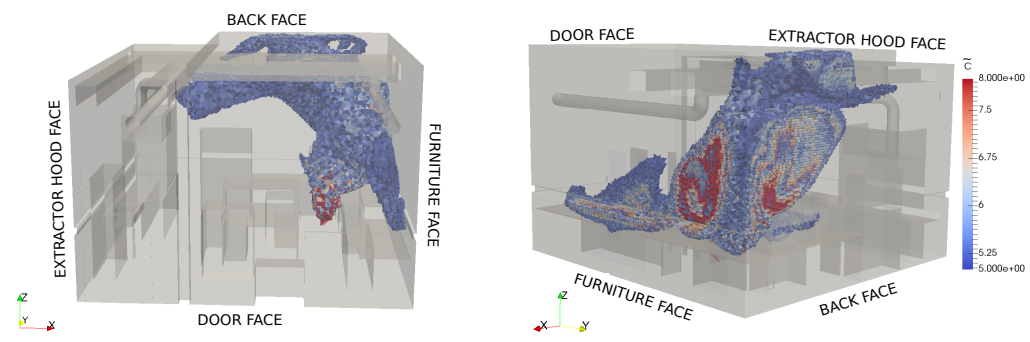

Figure 9: Simulation of the adjoint concentration $\tilde{C}$ associated with the optimal sensor position \#86

To quantify the observable on each lateral face, we use the observable 
criterion (8), taking into account the sensor features. Herein, we consider that the source emission to be detected has an amplitude of approximately $10 \mathrm{ppm}$ on a surface of approximately $0.25 \mathrm{~m}^{2}$ and that the limit of detection of the sensor is $0.01 \mathrm{ppm}$, i.e. $A_{s}=0.25 \mathrm{~m}^{2}, S=10 \mathrm{ppm}$ and $d I_{m}=0.01 \mathrm{ppm}$ in Eq. (8). The observable criterion (8) involving the ratio $A_{s} S / d I_{m}$, the observability results presented in the next paragraphs will be the same for other combinations of $A_{s}, S$ and $d I_{m}$, as long as $A_{s} S / d I_{m}=250$. For each potential sensor position, we compute the observable criterion and determine the observability on the four lateral walls. The highest observable area, which is approximately $7 \mathrm{~m}^{2}$, is obtained for Sensor position \#86. Consequently, Sensor \#86 is considered to be the optimal placement of the first sensor. In Figure 10, we use sectors to represent the sensor positions with "'acceptable observability", i.e. more than half of the maximum $7 \mathrm{~m}^{2}$ observability. Note that $15 \%$ (64 positions) of all the potential sensor positions satisfy this criterion. These results highlight the fact that haphazard placement of gas sensors may make it impossible to detect source emissions.

Figure 10 shows that most of the sensors are sensitive to source emissions on the furniture and back faces. Only 6 sensor positions (resp. 8 sensor positions) can cover a part of the extractor hood face (resp. the door face). We can see that the observable areas differ depending on the height of the sensor because the air flow is highly three-dimensional in an indoor environment.

After fixing Sensor \#86 as the first optimal sensor position, the proposed numerical strategy selects Sensors \#9, \#235 and \#268 as the optimal positions of four sensors to maximize the observable area on the wall faces. The optimal positions are represented by red circles in Figure 10, and the associated observability maps are shown in Figure 11. Sensors \#86 and \#9 are selected to cover both the furniture face and the back face. Neverthe- 
less, Sensor \#86 is sensitive to source emissions on the upper part of the furniture face, whereas Sensor \#9 can detect sources on the lower part. The sensitivity of the gas concentration at Sensor position \#9 to sources emitted from the lower part between the furniture and the wall is due to the air flow. Indeed, the air flow from the second ventilation grid passes under the furniture and licks the lower part of the furniture wall (see Figure 8). Then, the main part of the flow goes out from the corner of the furniture and the door faces, where Sensor \#9 is located. Sensor \#235 provides information for the extractor hood face and covers additional areas on the back face. Finally, the observability on the door face is provided by the fourth sensor, that is, Sensor \#268.

Let us consider Sensor positions \#86, \#9, \#235 and \#268 and study the influence of the LOD on the observability of the sources. The parameters $A_{s}$ and $S$ are kept at $0.25 \mathrm{~m}^{2}$ and $10 \mathrm{ppm}$. In Figure 11, we show the observable area associated with each sensor for a $10 \mathrm{ppb}$ LOD and for a 2 ppb LOD. As expected, a lower sensor LOD increases the observable area. At a 10 ppb LOD, Sensor \#86 can detect sources on a portion of the furniture and back faces. When the LOD is decreased to $2 \mathrm{ppb}$, sources can be detected on a larger surface of the furniture and back faces and on the door and extractor hood faces, which were not observable at a 10 ppb LOD. Similar results are obtained for Sensor \#235. Reducing the LOD to 2 ppb enables the observation of new surfaces, such as the door face. Nevertheless, we can see an exception for Sensor \#9. In this case, as the flow is confined between the wall and the furniture, the observable area is slightly increased for a $2 \mathrm{ppb}$ LOD compared to that for a $10 \mathrm{ppb}$ LOD. Finally, we observe that some wall areas are covered by multiple sensors when the LOD is $2 \mathrm{ppb}$.

In the last paragraph, we propose numerical validation of the observable 
criterion (8) in the 3D laboratory room. A $10 \mathrm{ppm}$ source positioned on the door face, as shown in Figure 11, is injected on a surface of $0.25 \mathrm{~m}^{2}$. From a direct simulation, we predict the gas dispersion in the laboratory room and obtain the concentrations at the different sensor positions. The results are given in Table 2 . The concentration is higher than $10 p p b$ only at Sensor position \#268. Hence, at a 10ppb LOD, the source can be detected by only Sensor \#86. In Figure 11, we can see that this result was correctly predicted by the proposed observable criterion when considering a 10ppb LOD. At a $2 p p b$ LOD, the observable criterion indicates that the source represented in Figure 11 can be detected by all the optimal sensor positions except Sensor \#9. The predicted result is validated by the gas concentration from the direct simulation reported in Table 2, showing that only the gas concentration at Sensor \#9 is below the $2 p p b$ LOD. Therefore, Sensor \#9 is not able to detect the source, completing the validation study.

\begin{tabular}{|c|c|c|c|}
\hline \multirow{2}{*}{ Sensor position number } & \multirow{2}{*}{$\begin{array}{c}\text { Concentration } \\
\text { at sensor position }\end{array}$} & $\begin{array}{r}\text { Source detected by the sensor ? } \\
\text { (Y: Yes, N: No) }\end{array}$ \\
\cline { 3 - 4 } & $3 p p b$ LOD & $10 p p b$ LOD \\
\hline$\# 86$ & $<1 p p b$ & $\mathbf{Y}$ & $\mathbf{N}$ \\
\hline$\# 9$ & $3 p p b$ & $\mathbf{Y}$ & $\mathbf{N}$ \\
\hline$\# 235$ & $49 p p b$ & $\mathbf{Y}$ & $\mathbf{N}$ \\
\hline$\# 268$ & & $\mathbf{Y}$ \\
\hline
\end{tabular}

Table 2: Numerical validation of the adjoint-based observable criterion in the 3D laboratory room - Concentration at sensor positions \#86, \#9, \#235 and \#268 simulated for the source location defined in Figure 11 and verification of the source detection for $2 p p b$ LOD sensors and 10ppb LOD sensors 


\section{Conclusions \& Prospects}

We proposed a CFD-based virtual testing strategy for the optimal placement of gas sensors to efficiently localize surface source emissions in indoor air quality assessment. This strategy relies on a criterion that integrates the adjoint framework and sensor features, such as the limit of detection, to evaluate, at a reasonable computation cost, the coverage area associated with different sensor positions. We considered the "optimal sensor placement" to be the combination of sensors that maximizes the coverage area. In the two studied applications, we showed that many potential sensor positions observe almost nothing and thus are unable to localize sources, which highlights the importance of using such sensor placement strategies. Then, we emphasized that the coverage area can be increased not only by adding sensors but also by using sensors with a lower limit of detection. Hence, when positioning indoor air quality devices, we have to consider both the limit of detection and the number of sensors. Finally, this work can be extended to the localization of sources emitted inside a defined volume, especially for outdoor air quality purposes.

\section{Acknowledgments}

This work was supported by the FUI 18 MIMESYS funded by Region Ile-de-France, which involves several partners: EcologicSense, TERA, ETHERA, FLUIDYN, CSTB, ESIEE Paris, and IFSTTAR. We also want to thank our colleagues Erick Merliot for the realization of the numerical mockup of the studied room and Rachida Chakir for the fruitful discussions about the direct simulations of the air flow and gas dispersion. 


\section{References}

[1] T. Hoang, R. Castorina, F. Gaspar, R. Maddalena, P. Jenkins, Q. Zhang, T. McKone, E. Benfenati, A. Shi, A. Bradman, Voc exposures in california early childhood education environments, Indoor Air 27 (3) (2017) 609-621.

[2] C. Godwin, S. Batterman, Indoor air quality in michigan schools, Indoor Air 17 (2) (2006) 109-121.

[3] N. Goodman, A. Wheeler, P. Paevere, P. Selleck, M. Cheng, A. Steinemann, Indoor volatile organic compounds at an australian university, Build. and Environ. 135 (2018) 344 - 351.

[4] D. Campagnolo, D. E. Saraga, A. Cattaneo, A. Spinazz, C. Mandin, R. Mabilia, E. Perreca, I. Sakellaris, N. Canha, V. G. Mihucz, T. Szigeti, G. Ventura, J. Madureira, E. de Oliveira Fernandes, Y. de Kluizenaar, E. Cornelissen, O. Hnninen, P. Carrer, P. Wolkoff, D. M. Cavallo, J. G. Bartzis, Vocs and aldehydes source identification in european office buildings - the officair study, Build. and Environ. 115 (2017) 18 - 24.

[5] A. Bari, W. Kindzierski, A. Wheeler, M.-E. Héroux, L. Wallace, Source apportionment of indoor and outdoor volatile organic compounds at homes in edmonton, canada, Build. and Environ. 90 (2015) 114 - 124.

[6] Indoor air quality (iaq), pollutants, their sources and concentration levels, Build. and Environ. 27 (3) (1992) 339 - 356.

[7] W. H. Organization, Selected pollutants, Tech. rep., WHO Regional Office for Europe (2010). 
[8] L. Morawska, P. K. Thai, X. Liu, A. Asumadu-Sakyi, G. Ayoko, A. Bartonova, A. Bedini, F. Chai, B. Christensen, M. Dunbabin, J. Gao, G. S. Hagler, R. Jayaratne, P. Kumar, A. K. Lau, P. K. Louie, M. Mazaheri, Z. Ning, N. Motta, B. Mullins, M. M. Rahman, Z. Ristovski, M. Shafiei, D. Tjondronegoro, D. Westerdahl, R. Williams, Applications of low-cost sensing technologies for air quality monitoring and exposure assessment: How far have they gone?, Environ. Int. 116 (2018) $286-299$.

[9] D. Bourdin, P. Mocho, V. Desauziers, H. Plaisance, Formaldehyde emission behavior of building materials: On-site measurements and modeling approach to predict indoor air pollution, J. of Hazard. Mater. 280 (2014) $164-173$.

[10] C. Dimitroulopoulou, M. Ashmore, M. Hill, M. Byrne, R. Kinnersley, Indair: A probabilistic model of indoor air pollution in uk homes, Atmos. Environ. 40 (33) (2006) 6362 - 6379.

[11] F. Haghighat, P. Fazio, T. Unny, A predictive stochastic model for indoor air quality, Build. and Environ. 23 (3) (1988) 195 - 201.

[12] W. Nazaroff, C. G., Mathematical modeling of chemically reactive pollutants in indoor air, Environ. Sci. \& Technol. 20 (9) (1986) 924 - 934.

[13] W. Yan, Y. Zhang, Y. Sun, D. Li, Experimental and cfd study of unsteady airborne pollutant transport within an aircraft cabin mock-up, Build. and Environ. 44 (1) (2009) $34-43$.

[14] G. Gan, H. B. Awbi, Numerical simulation of the indoor environment, Build. and Environ. 29 (4) (1994) 449 - 459. 
[15] X. Liu, Z. Zhai, Inverse modeling methods for indoor airborne pollutant tracking: literature review and fundamentals, Indoor Air 17 (6) (2007) $419-438$.

[16] X. Liu, Z. Zhai, Protecting a whole building from critical indoor contamination with optimal sensor network design and source identification methods, Build. and Environ. 44 (11) (2009) 2276 - 2283.

[17] A. D. Fontanini, U. Vaidya, B. Ganapathysubramanian, A methodology for optimal placement of sensors in enclosed environments: A dynamical systems approach, Build. and Environ. 100 (2016) 145 - 161.

[18] D. Papadimitriou, K. Giannakoglou, Computation of the hessian matrix in aerodynamic inverse design using continuous adjoint formulations, Comput. \& Fluids 37 (8) (2008) 1029 - 1039.

[19] J. Waeytens, P. Chatellier, F. Bourquin, Inverse computational fluid dynamics: influence of discretisation and model errors on flows in water network including junctions, ASME J. Fluids Eng. 137 (9) (2015) 17p.

[20] H. Elbern, H. Schmidt, O. Talagrand, A. Ebel, 4d-variational data assimilation with an adjoint air quality model for emission analysis, J. of Environ. Model. and Soft. 15 (2000) 539-548.

[21] J. Waeytens, P. Chatellier, F. Bourquin, Sensitivity of inverse advection-diffusion-reaction to sensor and control: a low computational cost tool, Comput. and Math. with Appl. 6 (66) (2013) 1082-1103.

[22] J. Waeytens, P. Chatellier, F. Bourquin, Impacts of discretisation error, flow modeling error and measurement noise on inverse transport- 
diffusion-reaction in a t-junction, ASME J. Fluids Eng. 139 (5) (2017) $10 \mathrm{p}$.

[23] J. Sykes, J. Wilson, R. Andrews, Sensitivity analysis for steady state groundwater flow using adjoint operators, Water Resour. Res. 3 (1985) $359-371$.

[24] F. Kauker, T. Kaminski, M. Karcher, M. Dowdall, J. Brown, A. Hosseini, P. Strand, Model analysis of worst place scenarios for nuclear accidents in the northern marine environment, J. of Environ. Model. and Soft. 77 (2016) 13-18.

[25] R. Becker, R. Rannacher, An optimal control approach to a posteriori error estimation in finite elements methods, Acta Numerica, Cambridge Press 10 (2001) 1-102.

[26] J. T. Oden, S. Prudhomme, Estimation of modeling error in computational mechanics, J. Comput. Phys. 182 (2002) 496-515.

[27] J. Waeytens, L. Chamoin, P. Ladevèze, Guaranteed error bounds on pointwise quantities of interest for transient viscodynamics problems, Comput. Mech. 49 (3) (2012) 291-307.

[28] V. Desauziers, D. Bourdin, P. Mocho, H. Plaisance, Innovative tools and modeling methodology for impact prediction and assessment of the contribution of materials on indoor air quality, Herit. Sci. 3 (1) (2015) 28.

[29] C. Wang, X. Yang, J. Guan, Z. Li, K. Gao, Source apportionment of volatile organic compounds (vocs) in aircraft cabins, Build. and Environ. 81 (2014) $1-6$. 
[30] B. Clarisse, A. Laurent, N. Seta, Y. L. Moullec, A. E. Hasnaoui, I. Momas, Indoor aldehydes: measurement of contamination levels and identification of their determinants in paris dwellings, Environ. Res. 92 (3) (2003) $245-253$.

[31] V. Akcelik, G. Biros, O. Ghattas, K. R. Long, B. van Bloemen Waanders, A variational finite element method for source inversion for convectivediffusive transport, Finite Elem. in Anal. and Des. 39 (8) (2003) $683-705$.

[32] K. Gurney, R. Law, A. Denning, P. Rayner, D. Baker, P. Bousquet, L. Bruhwiler, Y. Chen, P. Ciais, S. Fan, I. Fung, M. Gloor, M. Heimann, K. Higuchi, J. John, T. Maki, S. Maksyutov, K. Masarie, P. Peylin, M. Prather, B. Pak, J. Randerson, J. Sarmiento, S. Taguchi, T. Takahashi, C. Yuen, Towards robust regional estimates of CO2 sources and sinks using atmospheric transport models, Nature 415 (6872) (2002) 626-630.

[33] X. Liu, Z. Zhai, Location identification for indoor instantaneous point contaminant source by probability-based inverse Computational Fluid Dynamics modeling, Indoor Air 18 (1) (2008) 2-11.

[34] W. Liang, X. Yang, Indoor formaldehyde in real buildings: Emission source identification, overall emission rate estimation, concentration increase and decay patterns, Build. and Environ. 69 (2013) 114 - 120.

[35] A.-L. Pasanen, A. Korpi, J.-P. Kasanen, P. Pasanen, Critical aspects on the significance of microbial volatile metabolites as indoor air pollutants, Environ. Int. 24 (7) (1998) $703-712$. 
${ }_{640}^{6}$ [36] F. Archambeau, N. Méchitoua, M. Sakiz, Code Saturne: A Finite Vol${ }_{641}$ ume Code for the computation of turbulent incompressible flows - In642 dustrial Applications, Int. J. on Finite Vol. 1 (1).

${ }_{643}^{6}$ [37] T. J. Hughes, M. Mallet, M. Akira, A new finite element formulation 644 for computational fluid dynamics: Ii. beyond supg, Comput. Meth. in $645 \quad$ Appl. Mech. and Eng. 54 (3) (1986) $341-355$.

[38] F. Hecht, New development in freefem++, J. Numer. Math. 20 (3-4) 647 (2012) 251-265. 

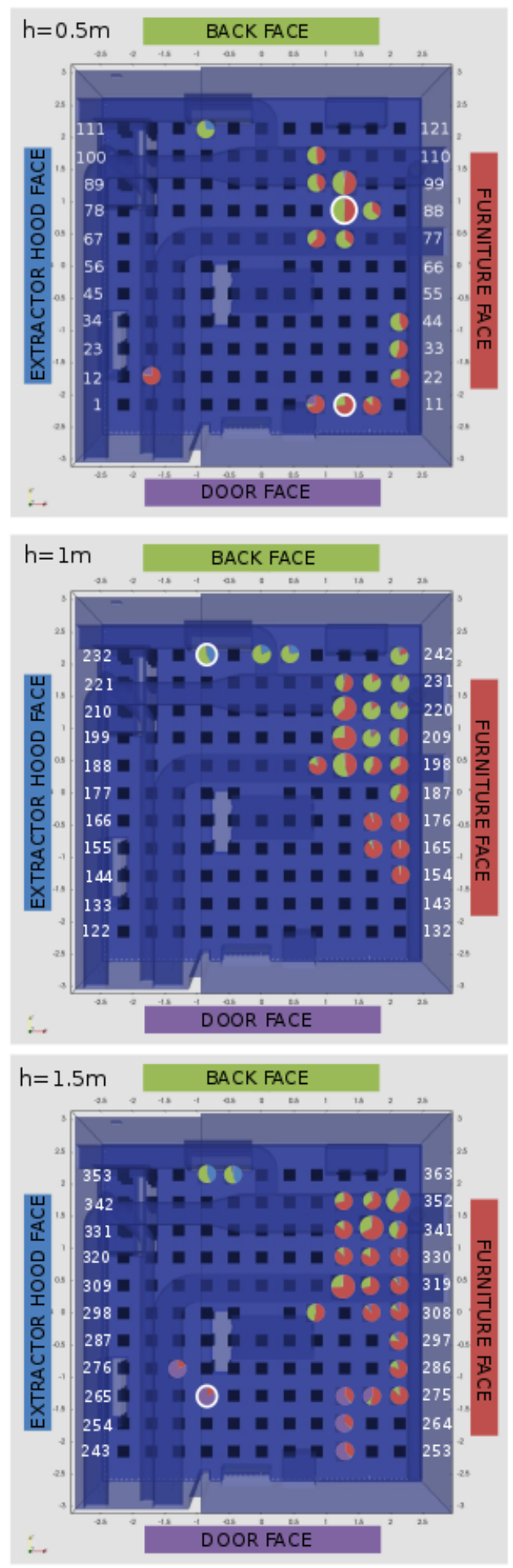

33

Figure 10: Potential positions of gas sensors at three levels $h=0.5 m, h=1 \mathrm{~m}$ and $h=1.5 \mathrm{~m}$ and observability representation by wall surface for sensors with an observable area more than half of the highest observability achieved by Sensor \#86 - Optimal sensor positions are shown as white circles 


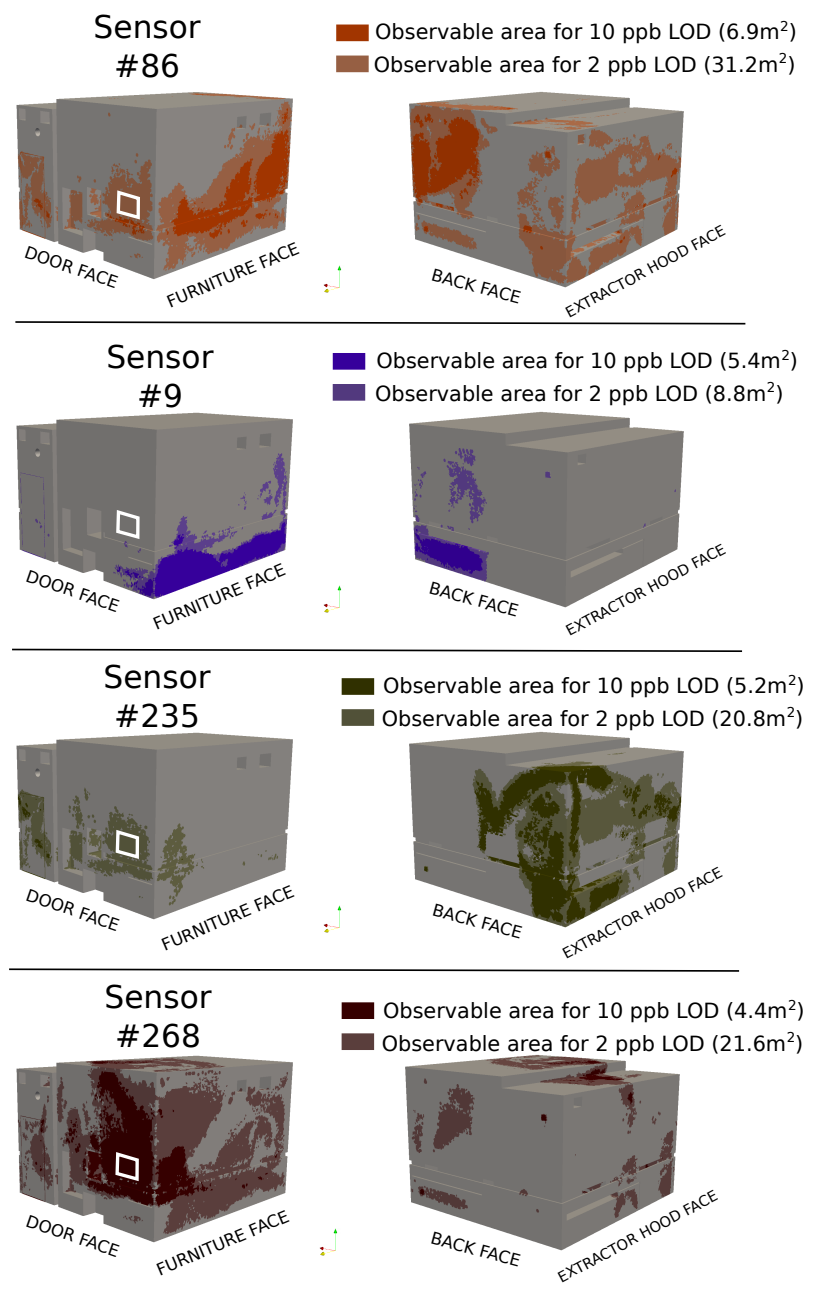

Figure 11: Map of the observable areas associated with gas sensors \#86, \#9, \#235 and \#268 at two different LODs (10 ppb and $2 \mathrm{ppb}$ ). The total observable areas are indicated in parentheses - Definition of a $0.5 m \times 0.5 m$ source (white square) for the numerical validation of the observability criterion 